Jean-Philippe Salier Sumiko Kurachi Kotoku Kurachi

\section{ADRESSES}

J.P. Salier: directeur de recherche ì l'Inserm. Inserm U.78, BP 73, 76233 Boisguillaume Cedex, France. S. Kurachi : assoriate investigator. K. Kurachi : professor. Department of human genetics, university of Michigan

\title{
Hémophilie B Leyden: les corrections naturelles d'un déficit temporaire de transcription
}

Des déficits enzymatiques sont très souvent observés malgré la présence de l'enzyme en concentration normale : la protéine est peu ou pas active car codée par un gène porteur d'une mutation ou d'une délétion dans une région exprimée. La grande majorité des hémophilies $B$ sont induites par des mutations de ce type dans le gène du facteur IX de la coagulation. Plus rarement, une mutation dans une région régulatrice d'un gène ou d'un transcrit aboutit à la synthèse d'une protéine normale et fonctionnelle, mais en quantité insuffisante. Enfin, le défaut de synthèse d'une protéine normale dans l'enfance peut se corriger secondairement au cours du développement de l'organisme, reflétant une situation exceptionnelle de "thérapie génique" naturelle. La maladie de Leyden, un cas rare d'hémophilie $B$, reste, jusqu'à présent, un exemple unique à cet égard. Cette affection résulte de mutations dans le promoteur du gène du facteur $\mathrm{IX}$, et comporte une réactivation de ce promoteur muté par des facteurs de transcription apparus à la puberté.

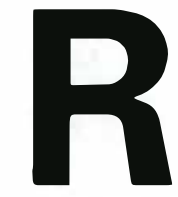

appelons très brièvement que les événements biochimiques qui suivent une atteinte vasculaire et aboutissent à l'hémostase, reposent sur l'activation de "facteurs ", qui sont en fait des enzymes plasmatiques initialement présentes sous forme de précurseurs inactifs. Ces enzymes activent d'autres précurseurs et l'ensemble de ces réactions en chaîne constitue la cascade de la coagulation. Les facteurs de coagulation VIII et IX sont essentiels dans cette cascade, et le dysfonctionnement ou, a fortion, l'absence de l'une ou l'autre de ces protéines provoque des anomalies de coagulation: ce sont les hémophilies "A" lorsque le facteur VIII est déficient, ou «B " lorsque le facteur IX est en cause. Les individus hémophiles ont un temps de saignement prolongé, évidemment dangereux en cas de blessure, et souffrent d'hémorragies internes spontanées, particulière- 
ment localisées aux articulations. Ces anomalies ne s'expriment qu'à l'état récessif, au moment où l'allèle intact n'est plus là pour synthétiser une protéine fonctionnelle. Effectivement, le gène du facteur IX (gène FIX) est situé sur le chromosome $\mathrm{X}$ (en position $\mathrm{Xq27}$ ). De ce fait, une mutation du gène $F I X$, responsable d'une hémophilie $B$, sera transmise par la mère, mais n'aura d'incidence pathologique que chez les descendants mâles, atteints à une fréquence de 1 pour 30000 par cette maladie. La plupart de ces mutations affectent les fonctions de l'un ou l'autre des nombreux domaines distincts du pré-pro-peptide précurseur du facteur IX, long de 461 acides aminés, et codé par les 8 exons du gène, lui-même s'étendant sur près de $40 \mathrm{~kb}$ [1]. Il est hors de propos de détailler ici la liste, toujours croissante, des mutations du gène FIX associées à une protéine anormale et à un phénotype hémophile. Le lecteur pourra, à ce propos, se reporter à un catalogue déjà long d'environ 800 mutations répertoriées, et régulièrement tenu à jour depuis 1990 par un comité ad hoc [2]. Dans le cadre de cette revue, notre intérêt va désormais se cantonner au champ plus étroit de la région d'ADN dite "promotrice" qui contrôle l'expression de ce gène, et des anomalies de fonctionnement de ce promoteur.

\section{Hémophilie B Leyden : tableau clinique et biologique}

La première observation d'une hémophilie $\mathrm{B}$ à caractère génétique et transitoire remonte à 1970 [3]. La description biologique et clinique de cette forme d'hémophilie $B$, dite "de Leyden ", souligne deux faits. D'une part, l'importance des anomalies de coagulation est corrélée à un déficit de concentration plasmatique du facteur IX, mesuré en tant qu'antigène, indiquant, dans ce cas, l'activité normale de la protéine synthétisée et donc l'absence d'une mutation affectant les régions codantes du gène FIX. D'autre part, la concentration plasmatique de cette protéine est peu ou pas mesurable chez le jeune garçon, puis croît en $\mathrm{m} / \mathrm{s} n^{\circ} 2$ vol. 10, fairier 94 moyenne de $4 \%$ à $5 \%$ par an à partir de l'âge de quatorze ans, pour finalement atteindre chez l'adulte un taux plasmatique se situant entre $30 \%$ et $70 \%$ de la normale. Parallèlement, les signes classiques d'hémophilie (hémarthrose, hématomes intra-musculaires) sont observés chez l'enfant mais disparaissent chez l'adulte [4]. Cet accroissement du taux plasmatique de facteur IX apparaissant à la puberté chez le garçon hémophile, et l'observation complémentaire qu'une augmentation analogue du taux de facteur IX n'est pas détectée chez les filles porteuses de la mutation, ont conduit à conclure que l'activité du gène FIX est sous la dépendance de la testostérone [4]. De fait, le traitement d'un garçon de huit ans par des dérivés de cette hormone a significativement augmenté son taux plasmatique de facteur IX [5]. I a conclusion atteinte dès 1985 [5] a donc été que, dans l'hémophilie B Leyden, une mutation du gène $F I X$ affecte la régulation de son expression, et non ses régions codantes.

\section{Le gène FIXLeyden et ses mutations}

Sur de telles bases, à partir de l'ADN de plusieurs malades montrant un phénotype Leyden, divers laboratoires ont cloné la région 5' flanquante du gène $F I X$, et comparé la séquence obtenue à celle du gène normal. Un bilan est présenté dans le Tableau I. Les mutations associées à cette maladie délimitent une courte région du gène $F I X$, appelée " région spécifique Leyden " par certains auteurs [10], couvrant au minimum les nucléotides -26 à +13 , et dans laquelle certains nucléotides peuvent présenter plusieurs mutations distinctes (Tableau I). médecine/sciences n'avait pas manqué, en temps voulu, de signaler l'existence de certaines de ces mutations et d'en souligner l'importance fonctionnelle $\left(\mathrm{m} / \mathrm{s} n^{\circ} 7\right.$, vol. 6, p. 710 et $n^{\circ} 7$, vol. 8, p. 739).

Le biais de sélection des individus inhérent à la recherche du phénotype Leyden ne permet évidemment pas de savoir si des mutations sans effet pathologique notable exis- tent à d'autres positions dans cette "région Leyden ". Toutefois, le nombre de cas d'hémophilie Leyden déjà étudiés permet de mettre en évidence la possibilité d'une variation de la gravité des signes cliniques et du taux plasmatique de facteur IX, en fonction de l'emplacement de la mutation observée. Le Tableau I illustre ce propos : la plupart des mutations en position -6 s'accompagnent d'un taux plasmatique de facteur IX relativement élevé, tant chez l'enfant qu'à l'âge adulte; en revanche, d'autres mutations, telle $A \rightarrow G$ en +13 , sont associées à un taux plasmatique plus bas. Enfin, un phénotype exceptionnel accompagne la mutation située en -26: le caractère transitoire de l'hémophilie a disparu, et le taux plasmatique de facteur IX reste nul à l'âge adulte. Siricto sensu, cette dernière mutation ne devrait donc pas être associée à l'appellation d'hémophilie B Leyden, dont le phénotype transitoire a, jusqu'ici, constitué le critère distinctif. Toutefois, cette mutation en -26 n'est que le cas extrême d'une série de mutations affectant plus ou moins sévèrement le fonctionnement du promoteur du gène FIX et, à ce titre, elle a donc toute sa place ici. En effet, ce sont bien les capacités transcriptionnelles - normales ou réduites - du gène FIX que nous allons envisager désormais.

\section{Un départ de transcription discuté}

L'emplacement du point de départ de la transcription du gène FIX normal est loin de faire l'unanimité. La figure 1 présente l'état actuel de la question. D'une part, elle met en évidence un désaccord concernant l'emplacement du principal point de départ de la transcription, situé en +1 ou en -176 selon les auteurs, et, dans ces deux cas, elle montre, d'autre part, une multiplicité de points de départ secondaires. En effet, lors de deux études distinctes $[19,20]$, toutes deux utilisant les techniques complémentaires d'allongement d'amorce et de protection de sonde contre la RNAse, le point de départ de transcription du gène FIX a tout d'abord été placé à la position +1 , ainsi que, avec une importance plus accessoire, aux posi- 


\section{RÉFÉRENCES}

1. Yoshitake S, Schach BC; Foster I)C: Davie EW, Kurachi $\mathrm{K}$ Nucleotide sequence of the gene for human factor IX (antihemophilic factor B). Biochemistry $1985 ; 24$ : 3736-50.

2. Giannelli F, Green PM, High KA, el al. Haemophilia B: database of point mutations and short additions and deletions, 4th edition, 1993. Nucleic Arids Res 1993; 21 3075-87.

3. Veltkamp IJ, Meilof J, Remmelts HC; Van der Vlerk D, Loeliger EA. Another genetic variant of haemophilia B: haemophilia B Leyden. Scand I Haematol 1970; 7: $82-90$.

4. Briēt E, Bertina RM, Van Tilburg NH, Veltkamp IJ. Hemophilia B Leyden. A sexlinked hereditary disorder that improves after puberty. $\dot{N} \mathrm{kingl} / \mathrm{Med}$ I982 ; 306 788-90

5. Briēt E, Wijnands MC;, Veltkamp IJ. The prophylactic treatment of hemophilia B leyden with anabolic steroids. Ann Interm Med 1985; 103: 225-6.

6. Crossley M, Iudwig M, Stowell KM, I) Vos P, Olek K, Brownlee (;). Recovery from hemophilia B Leyden : an androgenresponsive element in the factor IX promoter. Srience 1992 ; 257 : 377-9.

7. Reijnen MJ, Peerlinck K, Maasdam I, Bertina R.M, Reitsma PH. Hemophilia B Leyden: substitution of thymine for guanine at position -21 results in a disruption of a hepatocyte nuclear factor 4 binding site in the Factor IX promoter. Blood 1993; $82: 151-8$.

8. Reitsma PH, Bertina RM, Ploos van Amstel JK, Riemens A, Briēt E. The putative factor IX gene promoter in hemophilia B Leyden. Blood 1988; 72: 1074-6.

9. Chanem N, Costes B, Martin J, el al. Twenty four novel hemophilia B mutations revealed by rapid scanning of the whole factor IX gene in a French population sample. Eur J Hum Genet 1993; 1: 144-55.

10. Hirosawa S, Fahner JB, Salier JP, Wu C.T, Lovrien EW, Kurachi K. Structural and functional basis of the developmental regulation of human coagulation factor IX gene: factor IX Leyden. Proc Nall Acad Sci USA $1990 ; 87: 4421-5$. tions +4 et +30 . Soulignons que cette hétérogénéité possible du point de départ de transcription ne constitue pas une rareté pour un gène dépourvu de boîte TATA [20]. Mais, par la suite, le point de départ de transcription a été ré-examiné par les mêmes techniques, lors d'une étude fonctionnelle utilisant des constructions plasmidiques avec le gène de la chloramphénicol acétyl transférase (CAT) servant de gène test (gène rapporteur) [21]. Cette troisième étude n'a pas confirmé les précédentes observations puisque le point de départ a été alors localisé en position -150 , c'est-à-dire à 150 bases en amont de son précédent emplacement; trois autres points de départ mineurs ont également été observés entre -62 et -247 , mais aucun n'a été détecté à la position +1 [21]. Il a été avancé [20] que ces déterminations faites avec les constructions CAT étaient fausses, du fait même des constructions. Toutefois, cet argument ne tient plus, face aux tout récents résultats obtenus avec des $A R N m$ de foie humain: non seulement par la technique d'allongement d'amorce, mais aussi par reverse transcriptase-PCR, le principal point de départ de transcription est clairement localisé à -176 , même si un autre point de départ mineur est observé à -1 [22]. Enfin, et au vu de la bonne conservation du gène $F I X$ entre espèces [23], la description chez le chien d'un ADNc commençant à une position équivalente au nucléotide -175 chez l'homme [24] est un fort argument indirect en faveur d'un départ de transcription du gène humain en -176, c'est-à-dire bien plus en amont que le nucléotide +1 proposé initialement.

Un fait est certain: selon que le point de départ est en $+1[19,20]$ ou autour de $-150 /-180[21,22]$, la courte séquence génique allant de -26 à +13 et contenant toutes les mutations connues pour être associées à la maladie de Leyden, ou bien fait partie d'une région promotrice en grande partie non transcrite, ou bien est entièrement localisée sur une région transcrite (figure 1). Le second cas de figure ouvre alors des possibilités supplémentaires de régulation posttranscriptionnelle.

\section{Contrôle normal de transcription du gène FIX}

Jusqu’à un passé récent, les études structurales et fonctionnelles de la région régulatrice 5' flanquante du gène $F I X$ ont été très limitées. Ces études ont été menées sur le gène humain, par l'approche classique dans laquelle des constructions plasmidiques combinent un gène rapporteur CAT et des délétions orientées du promoteur du gène FIX [21]. Le facteur IX plasmatique est une protéine quantitativement mineure $(5 \mu \mathrm{g} / \mathrm{ml}$ chez l'adulte normal) et, si l'on admet la relation très simplifiée : taux de protéine $=\mathrm{f}$ (transcription), il n'est donc pas surprenant que ces études fonctionnelles aient montré une très faible activité transcriptionnelle intrinsèque du promoteur du gène FIX comparée, par exemple, à celle de promoteurs viraux [21]. Considérée dans son ensemble (figure 1), la région 5' régulatrice du gène FIX est tout d'abord constituée d'un promoteur minimal proximal, dépourvu de boîtes TATA et CCAAT évidentes, et situé dans la séquence -176 à -274 par rapport au point de départ de transcription (ici, et dans les paragraphes suivants, cette numérotation se réfère par commodité au point de départ utilisé dans la séquence "de référence" du gène FIX[1], mais ne préjuge pas de son exactitude). La région régulatrice comporte, de plus, un segment distal, en position -1400 à -1900 , qui a un effet négatif (silencer) sur le contrôle de la transcription, et peut, à ce titre, participer à l'activité faible de transcription du gène. Sur une partie de région 5' flanquante couvrant $7 \mathrm{~kb}$ au total, aucun autre élément régulateur distal n'a, jusqu'ici, été mis en évidence [21]. En outre, les constructions CAT concernant tant ce promoteur minimal que ce silencer ne montrent d'activité détectable que dans le contexte d'une lignée hépatocytaire, tel l'hépatome humain HepG2. Cette observation indique que l'expression du gène FIX est strictement limitée à l'hépatocyte. Ce résultat a, par ailleurs, été démontré lors de la recherche des transcrits FIX dans une large série 


\begin{tabular}{|c|c|c|c|c|c|}
\hline \multicolumn{6}{|c|}{ LES MUTATIONS DU GĖNE FIX ASSOCIÉES A L'HÉMOPHILIE LEYDEN } \\
\hline Point de mutation ${ }^{a}$ & Mutation & $\begin{array}{l}\text { Taux de FIX } \\
\text { avant } 14 \text { ans }\end{array}$ & $\begin{array}{l}\text { plasmatique }^{b} \\
\text { après } 20 \text { ans }\end{array}$ & $\begin{array}{l}\text { Origine de } \\
\text { la famille }\end{array}$ & Références \\
\hline $\begin{array}{l}-26 \\
-21 \\
-20 \\
-20 \\
-20 \\
-6 \\
-6 \\
-6 \\
-6 \\
-6 \\
-6 \\
-5 \\
-5 \\
+6 \\
+8 \\
+13 \\
+13 \\
+13 \\
+13\end{array}$ & $\begin{aligned} & G \rightarrow C \\
& T \rightarrow G \\
& T \rightarrow A \\
& T \rightarrow A \\
& T \rightarrow C \\
& G \rightarrow A \\
& G \rightarrow A \\
& G \rightarrow A \\
& G \rightarrow A \\
& G \rightarrow A \\
& G \rightarrow C \\
& A \rightarrow T \\
& A \rightarrow G \\
& T \rightarrow A \\
& T \rightarrow C \\
& A \rightarrow C \\
& A \rightarrow G \\
& A \rightarrow G \\
& \text { dél. } A\end{aligned}$ & $\begin{array}{c}0 \\
0 \\
1 \\
1 \\
9 \\
1 \\
11 \\
13 \\
9 \\
30 \\
<1 \\
3 \\
? \\
<2 \\
1 \\
<1 \\
12 \\
0 \\
1\end{array}$ & $\begin{array}{c}0 \\
15 \\
37 \\
60 \\
? \\
N \\
62 \\
70 \\
? \\
? \\
27 \\
? \\
? \\
>37 \\
32 \\
? \\
N \\
24 \\
40\end{array}$ & $\begin{array}{c}\text { Allemagne } \\
\text { Belgique } \\
\text { Hollande } \\
\text { Hollande? } \\
\text { France } \\
\text { France } \\
\text { USA } \\
\text { GB } \\
\text { France? } \\
\text { GB } \\
\text { France? } \\
? \\
\text { GB } \\
\text { USA? } \\
\text { GB } \\
\text { Allemagne? } \\
\text { France } \\
\text { Arménie } \\
\text { Grèce }\end{array}$ & $\begin{array}{l}{[6]} \\
{[7]} \\
{[8]} \\
{[2]} \\
{[9]} \\
{[9]} \\
{[10]} \\
{[11]} \\
{[12]} \\
{[13]} \\
{[12]} \\
{[14]} \\
{[15]} \\
{[16]} \\
{[17]} \\
{[2]} \\
{[9]} \\
{[18]} \\
{[18]}\end{array}$ \\
\hline
\end{tabular}

a Cette numérotation correspond à la séquence complète du gène FIX telle qu'elle a été initialement publiée [1] et prend en compte le point de départ de transcription numéroté $t 1$ dans cette même référence [1]. Cette numérotation ne tient donc pas compte du désaccord actuel concernant l'emplacement réel du point de départ de transcription, discuté par ailleurs dans cet article. b Activité de coagulation in vitro, exprimée en pourcentage de la normale. Lorsque le propositus n'a pas encore atteint la puberté et que des adultes ex-hémophiles de la même famille ont pû être examinés, les valeurs données représentent une moyenne familiale. $N$, valeur non précisée mais considérée comme normale.

c L'origine est indiquée avec une précision très variable selon les publications. L'ethnie n'est jamais précisée. Toutes les données présentées ici correspondent à des familles distinctes, sans liens de parenté connus. Les autres études familiales dans lesquelles la mutation n'a pas été totalement identifiée ne sont pas indiquées.

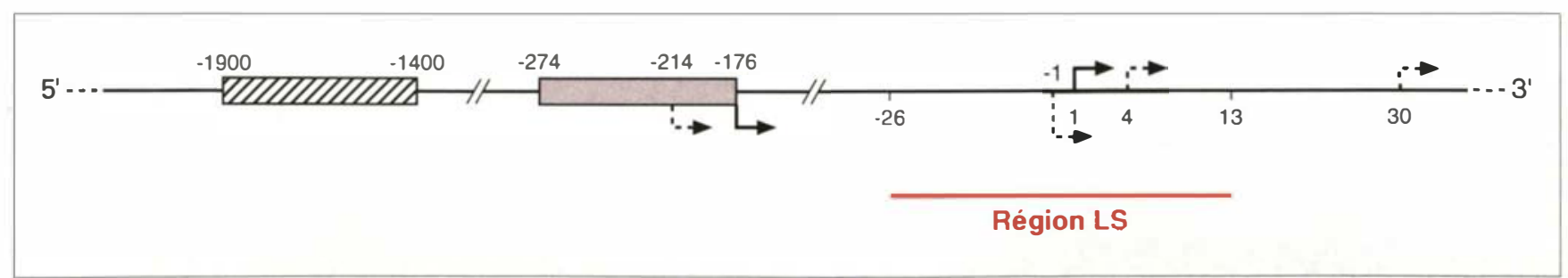

Figure 1. Positions relatives des points de départ de la transcription, du promoteur minimal, d'un silencer, et de la "région Leyden " dans le gène FIX humain. La région 5' flanquante du gène est représentée par une ligne continue. Les points de départ de transcription décrits dans la littérature sont indiqués par une flèche coudée ancrée au nucléotide au niveau duquel commence la transcription. Les points de départ majeurs sont en trait plein, ceux correspondant à des transcriptions mineures sont en pointillé. Les points de départ déterminés par Anson et Reijnen [19, 20] sont placés au-dessus du gène FIX; ceux déterminés Salier et Kurachi [21, 22] sont placés en dessous. Le promoteur minimal de -176 à -274 [21] est en bistre. Un élément de régulation négative couvrant la région -1400 à -1900 [21] est représenté par des hachures. La "région spécifique Leyden ", désignée par "LS" et délimitée par une ligne rouge, s'étend de -26 à +13. L'ATG correspondant le plus probablement à la méthionine d'initiation de la protéine facteur IX est en position +51 (absente sur ce schéma) [1]. La numérotation utilisée correspond à la séquence complète du gène FIX telle qu'elle a été initialement publiée [1] et elle se réfère au point de départ de la transcription numéroté +1 tel qu'il était connu à l'époque [19]. Les différents éléments ne sont pas tous représentés à la même échelle. 


\section{RÉFÉRENCES}

11. Crossley M, Winship PR, Austen DEG Riza CR, Brownlee GG. A less severe form of haemophilia B Leyden. Nucleic Acids Res $1990 ; 18$ : 4633

12. Vidaud D, Tartary M, Costa JM, et al. Nucleotide substitutions at the -6 position in the promoter region of the factor IX gene result in different severity of hemophilia B Leyden : consequences for genetic counseling. Hum Genet 1993; 91 : 241-4.

13. Stowell KM, Figueiredo MS, Brownlee GG, Jones P, Bolton-Maggs PHB. Haemophilia B Liverpool: a new British family with mild haemophilia B associated with a $-6 \mathrm{G}$ to A mutation in the factor IX promoter. Br J Haematol 1993; 85 : 188-90.

14. Picketts DJ, D’Souza C, Bridge PJ, Lil licrap $D$. An $A$ to $T$ transversion at position $\mathbf{- 5}$ of the factor IX promoter results in hemophilia B. Genomics 1992 ; 12 : 161-3.

15. Saad S, Green PM, Murday V, Minford A, Giannelli F. A novel promoter mutation in the factor IX gene. $\mathrm{Br}$ / Haematol 1992 80 (suppl 1): 16 .

16. Freedenberg DL, Black B. Altered developmental control of the factor IX gene: a new $\mathrm{T}$ to $\mathrm{A}$ mutation at position +6 of the FIX gene resulting in hemophilia B Leyden. Thromb Haemost 1991; 65 : 964.

17. Royle G, Van de Water NS, Berry E, Ockelford PA, Browett PJ. Haemophilia B Leyden arising de novo by point mutation in the putative factor IX promoter region. Br J Haematol 1991; 77 : 191-4.

18. Reitsma PH, Mandalaki T, Kasper CK Bertina RM, Briēt $\mathrm{E}$. Two novel point mutations correlate with an altered developmental expression of blood coagulation factor IX (hemophilia B Leyden phenotype). Blood 1989; 73 : 743-6.

19. Anson DS, Choo KH, Rees DJG, et al. The gene structure of human antihaemophiliac factor IX. EMBO J 1984; 3 : 1053-60. d'organes de primate, parmi lesquels seul le foie s'est avéré positif [21]. Enfin, le gène $F I X$ est soumis à un contrôle au cours du développement: une étude chez la souris montre que le taux hépatique des ARNm correspondants augmente significativement dans la période périnatale et atteint son maximum à l'âge adulte [25].

Il est maintenant bien connu que la région 5' flanquante de la plupart des gènes à expression préférentiellement ou strictement hépatique est sous la dépendance d'un ou de plusieurs facteurs de transcription appartenant à au moins quatre familles de protéines, dont les représentants principaux sont appelés respectivement HNF-1, HNF-3, HNF-4 et C/EBP [26]. En ce qui concerne le gène $F I X$, ce n'est que très récemment que l'importance de certains de ces facteurs, et l'existence des domaines de fixation correspondants sur la région promotrice du gène, viennent d'être appréciées, précisément lors des études portant sur l'hémophilie B Leyden.

\section{Perturbations de transcription dans le promoteur du gène FIX Leyden}

Étant donné la lourdeur des techniques mises en jeu lors d'une analyse fonctionnelle de promoteur de gène, il n'est pas surprenant que toutes les mutations connues pour être associées à un phénotype Leyden (Tableau I) n'aient pas systématiquement donné lieu à une telle étude. Quatre équipes distinctes se sont toutefois attaquées à cette tâche, chacune privilégiant certaines des mutations connues [6, 7, 10, 22, 27-29]. En outre, ces équipes ont focalisé leurs recherches sur le promoteur proprement dit, et par conséquent nous n'envisagerons pas ici l'importance d'éléments régulateurs distaux, tels que le silencer évoqué précédemment ou d'éventuels enhancers. Nous allons donc tout d'abord considérer mutation par mutation - les anomalies de fixation de facteurs de transcription sur le promoteur, et leurs conséquences. Le fait que les travaux des équipes concernées aient finalement abouti à des propositions diver- gentes de mécanismes compensatoires sera ensuite discuté. La figure 2 présente les sites de fixation (boites) de facteurs de transcription, mis en évidence sur la région promotrice du gène $F I X$. Au sein du promoteur normal, les différentes études s'accordent sur la présence de six boîtes majeures [22, 27, 29], numérotées ici I à VI, la boîte VI étant la plus distale. Par des techniques in vitro (empreinte à la DNAse 1, retard sur gel), ou in vivo (transfections de plasmides munis d'un gène rapporteur), les boîtes I et VI ont été clairement identifiées comme des sites de liaison fonctionnels pour la protéine $\mathrm{C} / \mathrm{EBP}$, la boîte III fixe le facteur HNF-4, et la boîte $\mathrm{V}$ fixe le facteur NF-1 [6, 22, $27,28]$. A ce jour, les facteurs aptes à se fixer sur les boîtes II et IV ne sont pas encore identifiés. Notons également que seules les boîtes I, II et III recouvrent la région LS (figure 2). L'importance de cette région LS dans le contrôle d'expression du gène $F I X$ a été initialement démontrée par des constructions $C A T$ sous contrôle du promoteur FIX plus ou moins délété en 3' dans sa région $\mathrm{LS}$ : la délétion de la boîte I, mais non celle des boîtes suivantes, permet de conserver à ce promoteur une activité de base normale [10]. Les analyses fonctionnelles des mutations naturelles ont ensuite précisé cette observation.

\section{Effet d'une mutation en position $+13$}

Une seule étude, relativement limitée [27], a jusqu'ici examiné l'importance fonctionnelle de cette mutation dans la boîte I. La mutation $\mathrm{A} \rightarrow \mathrm{G}$ en +13 abolit la capacité de la boîte I à lier le facteur C/EBP. Dans l'environnement d'une lignée d'hépatome humain, le promoteur muté associé à un gène CAT conserve une activité basale normale, mais il n'est plus transactivé par la protéine C/EBP. Cette étude [27] n'a toutefois pas considéré les éventuels rapports entre cette boîte I mutée et l'activation du promoteur par les androgènes. Par ailleurs, l'importance des autres mutations $(+6,+8)$ de cette boîte I reste encore à étudier en termes de perte de fonction. 
Effet d'une mutation en position -5 ou -6

Les positions -5 et -6 sont toutes deux localisées dans la boîte II (figure 2), et affectent très probablement de la même manière cette entité fonctionnelle. Par conséquent, nous analyserons globalement ici l'effet des mutations correspondantes. A la différence de la mutation +13 , les mutations en -5 ou -6 abolissent l'activité basale du promoteur [10,27, 29], ce qui a amené à suggérer que les mécanismes d'inactivation associés à la mutation +13 et à la mutation -6 pourraient être radicalement différents [27]. En accord avec cette idée, il a d'ailleurs été montré que - contrairement à la mutation $+13-$ les mutations -5 ou -6 dans la boîte II n'affectent pas la fixation de C/EBP sur la boîte I [27, 29]. Mais, par ailleurs, la mutation -5 abolit sur la boîte II la fixation d'un facteur de transcription non identifié [29], indiqué "X" dans la figure 2. En outre, cette mutation réduit d'un facteur 2 à 3 la réponse d'une construction promoteur/CAT à une induction androgénique [10].

Chez les mutants -5 ou -6 , la capacité d'une construction promoteur/CAT d'être transactivée par la protéine C/EBP est abolie [27, 29]. Toutefois, un promoteur mutant -5 peut être transactivé par $\mathrm{C} / \mathrm{EBP}$ en présence d'un autre facteur de transcription, DBP, agissant en synergie avec $\mathrm{C} / \mathrm{EBP}$; la fixation de $\mathrm{C}$ /EBP sur sa cible est alors grande- ment améliorée par cette interaction transitoire [29]. Cette synergie $\mathrm{C} / \mathrm{EBP}(\mathrm{DBP})$ requererait un site de liaison de faible affinité pour $\mathrm{C} / \mathrm{EBP}$ et n'existerait pas dans le promoteur normal [29]. L'apparition du facteur DBP a lieu à la puberté, tout au moins chez le rat. ll n'en a pas fallu plus pour proposer chez l'homme, à partir de la puberté, un mécanisme de réactivation du promoteur Leyden muté sous l'effet de C/EBP en synergie avec DBP [29]. Toutefois, les critiques à cette élégante hypothèse sont multiples. Plus particulièrement, on doit regretter l'absence d'une démonstration rigoureuse de l'implication de la boîte II dans la fixation de C/EBP(DBP), qui a, en fait, la boîte V et surtout la boîte VI pour

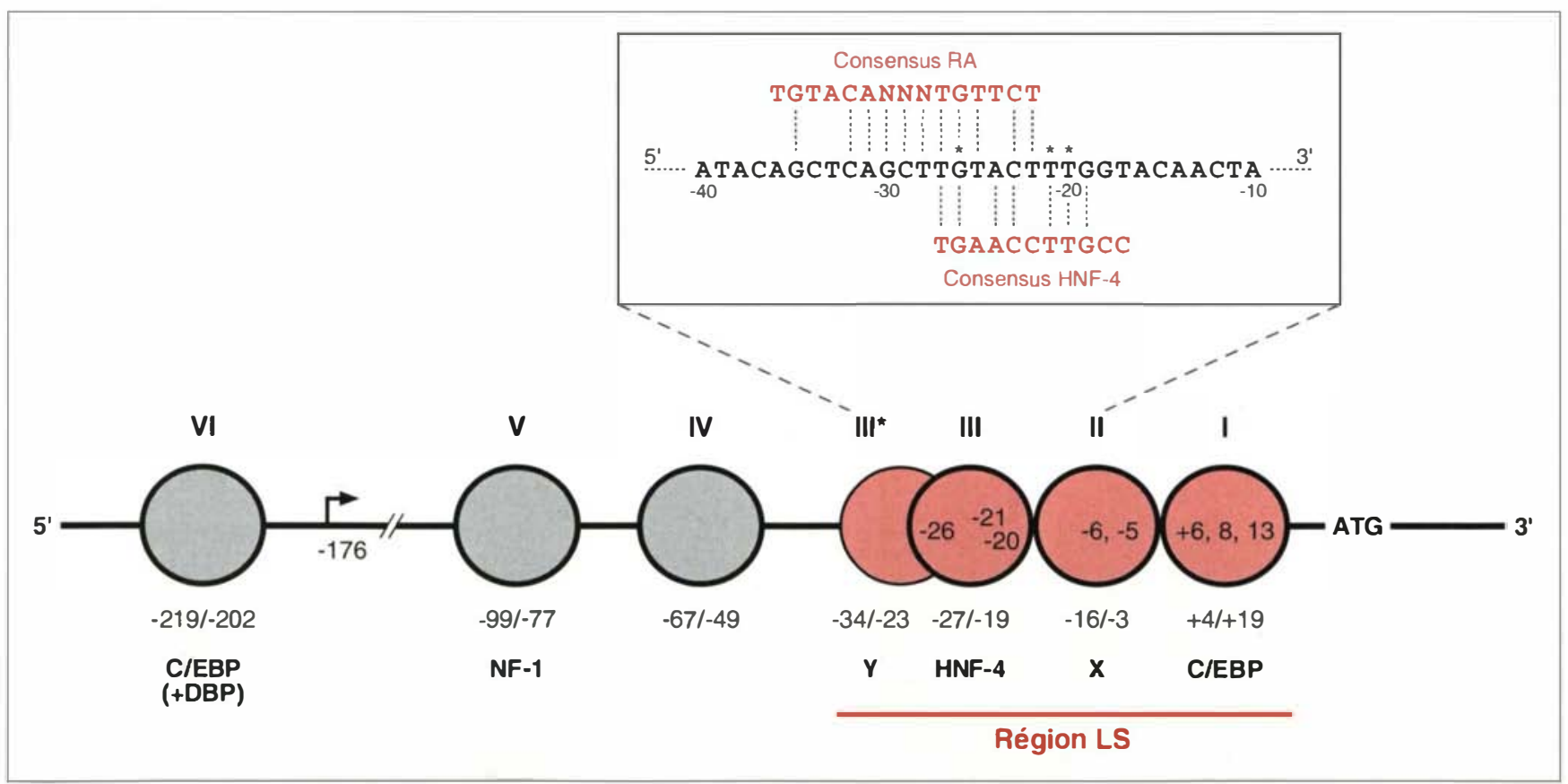

Figure 2. La «région Leyden " du gène FIX humain, et son environnement. La région $5^{\prime}$ flanquante du gène est représentée par une ligne continue. La "région spécifique Leyden" est désignée par "LS", et délimitée par une ligne rouge. La probabilité d'un point de départ principal de transcription en -176 est indiquée. Chacune des régions d'ADN ("boîtes") aptes à fixer un ou des facteurs de transcription est indiquée par un cercle numéroté de I à VI. Une boîte supplémentaire, notée III', n'apparaît que lors d'une mutation Leyden en position -20. Les limites de chaque boîte, déterminées par la technique d'empreinte à la DNAse l, et/ou par homologie de séquence, sont indiquées sous la boîte par la numérotation nucléotidique correspondante. Les emplacements des mutations associées à l'hémophilie Leyden sont indiqués dans les boîtes. Lorsque la fixation d'un (ou plusieurs) facteur(s) de transcription sur une boîte est bien établie, le nom de ce(s) facteur(s) est indiqué sous la boîte. $X$ et $Y$ sont deux facteurs de transcription encore non identifiés. La séquence de la région -10 à -40 est agrandie pour montrer dans la boîte III/II' le chevauchement d'une séquence (-17 à -27) de fixation de HNF-4 et d'une séquence (-22 à -36) de fixation d'un récepteur des androgènes (ou du facteur Y qui en dépend). Les deux séquences nucléotidiques consensus de fixation de HNF-4 et du récepteur des androgènes (RA) sont également indiquées. Dans la région considérée du promoteur FIX, les mutations connues sont indiquées par des étoiles. 


\section{RÉFÉRENCES}

20. Reijnen MJ, Bertina RM, Reitsma PH. Localization of transcription initiation sites in the human coagulation factor IX gene FEBS I ell 1990) ; 270: 207-10.

21. Salier JP, Hirosawa S, Kurachi K Func tional characterization of the $5^{\prime}$ regulatory region of human factor IX gene. I Biol Chem 1990; 265 : 7062-8.

22. Kurachi S, Furukawa M, Salier JP, el al. Regulatory mechanism of human factor IX gene: protein binding at the Leyden-specific region. Biochemistry 1994 (sous presse).

23. Pang CP, Crossley M, Kent (;, Brownlee G(;. Comparative sequence analysis of mammalian factor IX promoters. Nucleic Acids Res 1990; 18: 6731-2.

24. Evans JP, Watzke HH, Ware JL, Stafford DW, High KA Molecular cloning of a cDNA encoding canine factor IX. Blood 1989 ; 74 : 207-12.

25. Yao SN, De Silva AH, Kurachi S, Samuelson L., Kurachi K. Characterization of a mouse factor IX cDNA and developmental regulation of the factor IX gene expression in liver. Thromb Haemost 1991 $65: 52-8$.

26. Ciliberto (', Colantuoni I', De Francesco R, et al. Transcriptional control of gene expression in hepatic cells. In : Kano M. General and cell-cypespecific gene expression. Boston: Bukhauser, 1993 (sous presse).

27. Crossley M, Brownlee G(;. Disruption of a $\mathrm{C} / \mathrm{EBP}$ binding site in the factor IX promoter is associated with haemophilia B. Nature 1990; 345 : 444-6.

28. Reijnen MJ, Sladek FM, Bertina RM, Reitsma PH. Disruption of a binding site for hepatocyte nuclear factor 4 results in hemophilia B Leyden. Proc Natl Acad Sci USA 1992; 89: 630()-3.

29. Picketts DJ, Lillicrap DP, Mueller CR. Synergy between transcription factors DBP and C/EBP compensates for a haemophilia B Leyden factor IX mutation. Nature Genet 1993; 3 : 175-9.

30. Theil EC. Regulation of ferritin and transferrin receptor mRNAs. J Biol C.hem $1990 ; 265: 4771-4$ cibles [29]. En effet, dans une analyse in vitro de retard sur gel, les boîtes $\mathrm{V}$ et I'T mais non la boîte II fixent $\mathrm{C} / \mathrm{EBP}$, et cette fixation est très fortement amplifiée en présence de DBP [29]. Si ces boîtes V et VI sont les cibles réelles de l'activation postpubertaire de C/EBP par DBP, alors pourquoi existe-t-il une mutation Leyden (mutation en -26 sur la boîte III) qui n'est pas compensée à la puberté ? En outre, la démonstration, à l'aide de constructions $C A T$, d'une absence de synergie C/EBP(DBP) dans le promoteur normal dépend de la précision dans les mesures d'activité $C A T$, dont les écarts-types, importants dans la publication considérée [29], ont pu masquer par inadvertance une telle synergie. Si tel est le cas, la synergie $\mathrm{C} / \mathrm{EBP}(\mathrm{DBP})$ et la fixation de C/EBP sur les boîtes $V$ et VI seraient alors... un phénomène physiologique, indépendant de la maladie de Leyden! En fait, les auteurs eux-mêmes [29] n'ont pu éviter d'envisager que l'effet de $\mathrm{C} / \mathrm{EBP}(\mathrm{DBP})$ serait observable à condition que l'activité du promoteur soit affaiblie, ce qui n'exclut donc pas un tel effet sur le promoteur normal. Ainsi, et en dépit de l'interaction C/EBP(DBP) sur la boîte VT, le facteur $\mathrm{X}$, inapte à se fixer sur la boîte II mutée, et jusqu'alors non identifié (voir plus haut) mérite toute notre attention dans le contexte de l'hémophilie Leyden.

\section{Effet d'une mutation en position -20 ou -21}

L'importance fonctionnelle des mutations en -20 ou -21 , toutes deux sur la boîte III, a été particulièrement étudiée $[6,7,10,22,28]$. La séquence normale -17 à -27 dans la boîte III est indiscutablement la cible du facteur HNF-4, dont la fixation est abolie en présence d'une mutation Leyden en -20 ou -21 . Lorsqu'elle contient une telle mutation, une construction promoteur/CAT perd toute activité basale, et n'est plus transactivable par HNF-4 $([6,7,28]$, $\mathrm{m} / \mathrm{s} n^{\circ} 7$, vol. 8, p. 739).

De façon tout à fait remarquable, dans le promoteur normal, la séquence nucléotidique -22 à -36 qui recouvre partiellement le site de fixation de HNF-4 dans la boîte III, est, dans une certaine mesure, similaire à la séquence consensus de reconnaissance du récepteur nucléaire des androgènes, comme cela est détaillé dans la figure 2 . Une activité, réduite, certes, de fixation du récepteur nucléaire des androgènes par cette séquence -22 à -36 a été montrée par constructions CAT et par retard sur gel [6]. Dans le cas d'une mutation en -20 ou -21 , ainsi se retrouve-t-on dans une situation où la fixation de HNF-4 est abolie en un premier site, qui lui-même chevauche un site de fixation du récepteur nucléaire des androgènes, ce second site n'incluant pas la mutation (figure 2). Une mutation en position -20 diminue, mais ne supprime pas, la réponse aux hormones androgènes d'une construction promoteur/CAT $[6,10]$ et la séquence -22 à -36 conserve sa capacité de fixer le récepteur nucléaire des androgènes [6, 7]. Sur de telles bases, un mécanisme compensatoire aboutissant à la transcription postpubertaire dans l'hémophilie Leyden a bien entendu été proposé [6] : alors que le promoteur normal fonctionne sous l'effet de HNF-4 fixé sur la boîte III, la mutation -20 de cette boîte III abolit la fixation de HNF-4, qui laisse ainsi la place libre pour une fixation du récepteur nucléaire des androgènes sur le site contigu -22 à -36. A partir de la puberté, la testostérone active le récepteur nucléaire des androgènes qui se fixe alors sur son site et déclenche la transcription du promoteur FIX I eyden. Ce mécanisme élégant, détaillé dans la figure 2, a été également pris en compte lors des études portant sur la mutation -21 [7].

En accord général avec ce point de vue, il a été très récemment montré par la technique d'empreinte à la DNAse I que, chez le mutant -20, l'empreinte $-19 /-27$, normalement associée à la fixation de HNF-4, est remplacée par une empreinte décalée -23/-34 (boîte III' dans la figure 2) correspondant à la fixation d'un facteur de transcription non identifié [22] désigné par "Y" dans la figure 2. Toutefois, les mêmes auteurs ont montré que la protéine Y, responsable de l'empreinte en III', est présente dans des lignées cellulaires (BHK, CV1) qui n'expri- 
ment pas le récepteur nucléaire des androgènes, et que $Y$ n'est pas reconnu par un anticorps dirigé contre ce récepteur. Ces éléments excluent par conséquent que le récepteur nucléaire des androgènes et Y soient une même entité [22]. Enfin, la quantité de protéine Y est multipliée par un facteur 3 dans des cellules d'hépatome HepG2 cultivées en présence de testostérone [22]. Ainsi, dans le cas de la mutation -20 , c'est la protéine $\mathrm{Y}$ - et non le récepteur nucléaire des androgènes - qui déclenche la transcription sous l'effet de la testostérone associée à la puberté.

\section{Effet d'une mutation en position $-26$}

Étant donné le phénotype inhabituel qui lui est associé (absence d'activation du gène $F I X^{1}$ inden à la puberté), la mutation -26 a provoqué un intérêt tout particulier $\left(m / s n^{\circ} 7\right.$, vol. 8 , p. 739). Cette mutation, située elle aussi sur la boîte III, a généralement été étudiée de manière concomitante avec les mutations -20 ou -21 de la même boîte $[6,7,22,28]$. La mutation -26 abolit, elle aussi, la fixation de HNF-4 sur la boîte III $[6,28]$, et une construction promoteur/CAT n'est pas transactivable par ce facteur [28]. En outre, la faible capacité de fixation de récepteur nucléaire des androgènes, typique de la séquence -22 à -36 normale, est totalement supprimée par la mutation $-26[6,7]$. Ainsi cette mutation -26 est-elle responsable de l'absence de fixation de HNF-4 sur la séquence -17 à -27 , et de l'absence de fixation du récepteur nucléaire des androgènes (ou de la protéine Y mentionnée ci-dessus) sur la séquence -22 à -36 . Et ainsi s'expliquerait, dans ce cas particulier, l'inactivité du gène $F I X^{\top \text { roden }}$ avant et après la puberté.

Qu'en est-il des interactions à distance entre les éléments cis et les facteurs trans?

Jusqu'à présent, et par commodité de présentation, nous avons envisagé séparément chacune des mutations et des boîtes le long de la région LS, et de même avons-nous fait en ce qui concerne le(s) facteur(s) nucléaire(s) sur la boîte affectée par la mutation. On ne peut toutefois pas ignorer les modulations de la transcription dues à des coopérations de fixation ou d'activation, résultant d'interactions entre facteurs [26]. Ce domaine d'étude, bien qu'en plein essor, n'a guère été pris en compte en ce qui concerne le promoteur du gène FIX ${ }^{\text {erden }}$. Dans ce dernier cas, toutefois, l'interaction C/EBP-DBP sur les boîtes V et VI lors d'une mutation -5 de la boîte II [29] et la possibilité d'une disparition de l'empreinte de la boîte II lorsque la boîte III est mutée en -20 [22] illustrent le fait que des mécanismes d'interactions encore ignorés peuvent affecter la région LS.

\section{Le promoteur du gène FIXLeyden à la puberté : une correction ou des corrections?}

En dépit des différences entre les mécanismes compensatoires proposés pour les diverses mutations Leyden, plusieurs des publications qui ont découlé de ces travaux ont tenté d'élargir à l'ensemble des mutations le mécanisme associé à la mutation spécifiquement étudiée. Toutefois, si l'on veut bien sortir du cadre contraignant des discussions d'articles, où l'académisme scientifique implique de montrer à tout prix la portée et la généralité d'une observation, il convient maintenant de se demander si, réellement, un même mécanisme correctif s'applique à toutes les mutations Leyden. Notons tout d'abord que des mécanismes différents évoquent immédiatement la possibilité d'un phénotype de gravité variable selon la mutation. De ce point de vue, les données du Tableau I, pour limitées qu'elles soient, suggèrent que les mutations sur la boîte Il, c'est-à-dire en -5 ou -6 , induisent une gravité moindre de l'hémophilie $\mathrm{B}$.

L'effet de la testostérone dans le "réveil" du gène $F I X^{l \text { egden }}$ ne semble guère discutable lorsque l'on considére l'amélioration des symptômes, à la fois postpubertaire, progressive, et parallèle à l'augmentation du taux de testostérone circulante. Certes, une critique qui a pu être faite du(des) mécanisme(s) expliquant l'amélioration de transcription du gène $F I X^{\text {exden }}$ par la testostérone, réside dans le très modeste accroissement de réponse - au mieux un facteur 2 à 3 - du promoteur normal sous l'action des androgènes, effet dont l'amplitude peut difficilement être comparée à l'augmentation significative de transcription du gène $F I X^{7_{e y d e n}}$ après la puberté. Doit-on alors envisager qu'une analyse trop réductionniste du gène FIX normal, dans le cas présent limité à son seul promoteur, n'a pu prendre en compte des interactions essentielles de ce promoteur avec son environnement nucléotidique naturel, ce dernier incluant des éléments de réponse aux hormones pour l'instant ignorés? Ou bien, doit-on plutôt considérer que l'effet modéré de la testostérone ne concerne que le seul gène muté, et initialement inactif? La fixation physiologique de HNF-4 sur la boîte III normale, qui empêche le fonctionnement de la séquence voisine et accessoire, ellemême cible du récepteur nucléaire des androgènes ou de $\mathrm{Y}$, va tout à fait dans ce sens. En tout cas, au moins en ce qui concerne cette boîte III, le mécanisme général impliquant un effet direct de la testostérone, et expliquant la présence ou l'absence de correction associée aux mutations $-20,-21,-26$, semble peu discutable. Pour autant, lors de l'amélioration post-pubertaire, la testostérone est-elle dans tous les cas responsable d'un effet direct sur le promoteur, quelle que soit la mutation considérée? Dans le cas de la mutation +13 de la boîte I, le rôle correcteur de la testostérone, bien que soupçonné et semblant influencer la fixation de facteurs de transcription sur cette boîte, reste pour l'instant inexpliqué [22]. Et surtout, la mutation -6 sur la boîte II semble réduire, certes, la réponse d'une construction promoteur/CAT à une induction par les hormones androgènes [10], mais la fixation de facteurs nucléaires sur la boîte II semble indépendante des androgènes [22]. Or il se trouve que c'est précisément la boîte II, et les mutations associées en -5 et -6 , qui sont impliquées dans un mécanisme correcteur tout à fait original passant, 
non plus par la voie du facteur " récepteur des androgènes ", mais - on l'a vu — par celle d'une autre protéine nucléaire, DBP, dont la concentration est contrôlée au cours du développement [29]. Dans ce dernier cas, que la synthèse de DBP soit elle-même liée ou non à l'apparition des androgènes, ces derniers ne seraient plus responsables d'un mécanisme compensatoire direct au niveau du promoteur du gène FIX. Notons également que, étant donné ce mécanisme correctif associé à l'apparition de DBP, on peut imaginer que les filles porteuses d'une mutation en -5 ou -6 ont un accroissement du taux de facteur IX plasmatique à la puberté. Cette possibilité reste toutefois entièrement à explorer.

Enfin, rappelons ici l'importance du point de départ de la transcription qui - selon les équipes - est placé soit très en amont de la région LS, soit à l'intérieur de celle-ci (figure 1). Dans le second cas de figure, les boîtes II à VI font partie intégrante d'un promoteur. Mais si - comme nous le pensons - le point de départ de transcription est plus en amont qu'initialement proposé, les boîtes I à $\mathrm{V}$ sont alors transcrites (figure 2), situation qui donnerait éventuellement accès à des niveaux supplémentaires de régulation par fixation de facteurs nucléaires sur le transcrit. Une telle régulation existe pour d'autres ARN, tels ceux de la ferritine et du récepteur de la transferrine, dont la stabilité ou la traduction sont contrôlées par fixation d'une protéine sur le transcrit, au niveau d'un élément de réponse aux métaux ([30], $m / s \quad n^{\circ} 10$, vol. 9, p. 1145) De même, des études très préliminaires de la région LS du gène FIX ont montré une fixation de facteurs nucléaires sur des oligonucléotides simple-brin [22], mais la signification physiologique de cette observation reste à établir.

Ainsi arrive-t-on à conclure que, sur une longueur aussi réduite que le segment LS du promoteur du gène FIX, soit 40 nucléotides environ, plusieurs mécanismes indépendants, impliquant des facteurs de transcription très divers, peuvent aboutir aux corrections naturelles d'une trans- dérer que cette multiplicité des voies de correction est indispensable dans la seule hémophilie $B$ reviendrait à conférer au gène FIX une importance démesurée et à la protéine correspondante un rôle biologique crucial. L'existence d'une majorité de formes d'hémophilie $B$ dans lesquelles l'anomalie génétique reste sans recours, prouve qu'il n'en est rien. Il faut plutôt admettre que des études ayant, par chance, associé une maladie n'engageant pas le pronostic vital, un phénotype aisément quantifiable, un gène accessible en termes de séquence et de sondes, et un intérêt particulièrement marqué de nombreux laboratoires pour les facteurs de transcriptions hépatiques, ont fini par révéler, pour la première fois, au travers d'une maladie pourtant rare, des niveaux " annexes» de régulation de transcription, dépendant de facteurs synthétisés de manière discontinue dans le temps. L'importance de telles régulations palliatives pourrait bien, à l'avenir, se vérifier dans d'autres maladies

\section{Remerciements}

Nous sommes redevables à Alphonse Le Cam et François Tronche pour une lecture critique du manuscrit. Les recherches effectuées dans les laboratoires des auteurs ont été en partie financées par les National Institute of Health (attributions HL39099 et HL38644), le ministère de l'Enseignement Supérieur et de la Recherche, I'Inserm, et l'Université de Rouen (France)

\section{Summary}

Hemophilia B Leyden, natural rescues of a transient deficiency in transcription

An enzyme deficiency where a normal level of an abnormal pro tein is accounted for by a mutation within a coding region of the corresponding gene is conmon place in human pathology. Thus, hemophilia B which involves mutations in the coagulation factor IX gene, most of ten results from deleterious amino-acid changes in the protein sequence. In other, more limited, instances, an enzyme deficiency is accounted for by a mutation in a regulatory region of a gene or a noncoding region of its transcript. These mutations eventually result in a poor transcription or translation efficiency which is responsible for a low level of a normal protein. A further situation involves a unique step where a weakened transcription, induced by a mutation in a regulatory region of a gene, is secondarily restored to a (sub) normal level by the late appearance of developmentassociated transcription factor(s). This is examplified by the socalled Leyden phenotype, a rare case of transient haemophilia B. The patients suffering from this disease first present with bleeding disorders and a marked deficiency of a normal factor IX in plasma. However, after puberty they gradually recover from the disease as the plasma factor IX level increases to a sub-normal level. A testosterone-induced triggering of a mutated promoter in the factor IX gene accounts for this phenotype.

\section{TIRÉS A PART}

\section{J.P. Salier.}

